\title{
Internet e educação ao paciente
}

\section{Internet and education for the patient}

\author{
Bárbara Guimarães Bastos', Deborah Viviane Ferrari². \\ 1) Fonoaudióloga graduada pela Faculdade de Odontologia de Bauru - Universidade de São Paulo (FOB/USP). Mestranda em Fonoaudiologia - FOB/USP. \\ 2) Doutora em Neurociências e Comportamento - Instituto de Psicologia da Universidade de São Paulo. Professora Doutora do Departamento de Fonoaudiologia da \\ Faculdade de Odontologia de Bauru - Universidade de São Paulo. \\ Instituição: Departamento de Fonoaudiologia - Faculdade de Odontologia de Bauru - Universidade de São Paulo (FOB/USP). \\ Bauru / SP - Brasil \\ Endereço para correspondência: Deborah Viviane Ferrari - Departamento de Fonoaudiologia - FOB/USP - Alameda Octavio Pinheiro Brisola, 9-75 - Vila Universitária \\ - Bauru/SP - Brasil - CEP: 17012-901 - Telefone: (+55 14) 3235-8232 - E-mail: deborahferrari@usp.br \\ Fonte de auxílio à pesquisa: Fundação de Amparo à Pesquisa do Estado de São Paulo - Fapesp. Processo: 2008/07963-0 \\ Artigo recebido em 1º de Fevereiro de 2011. Artigo aprovado em 21 de Maio de 2011.
}

\section{RESUMO}

Introdução: O processo de educação ao paciente tem como objetivos melhorar o conhecimento e habilidade do paciente e/ou família de forma a influenciar as atitudes e comportamentos necessários para manter ou melhorar a saúde. Deve ser uma parte integral da comunicação entre profissionais de saúde e pacientes, podendo isto ocorrer por meio da comunicação interpessoal e outros diferentes meios como panfletos, manuais e, mais recentemente, recursos computacionais. Objetivo: Este artigo de atualização aborda a educação ao paciente e as potencialidades da internet para este processo, apresentando ainda algumas iniciativas na área da audiologia. Discussão: As informações relacionadas à saúde são populares na internet, e incluem websites interativos, portais, emails, aplicações de telessaúde, entre outros. A educação de pacientes apoiada pela internet pode contribuir para solucionar um grande desafio ético, político e econômico: o problema de conciliar as necessidades e expectativas dos pacientes com as características e limitações do sistema de saúde. Embora a utilização da internet para a educação ao paciente seja promissora, esta não é uma solução que deve ser utilizada sem planejamento cuidadoso, monitoramento e avaliação. $\mathrm{Na}$ audiologia existem poucas iniciativas com o uso de e-mails e websites para complementação da educação ao paciente.

Considerações Finais: Os profissionais da saúde, incluindo os fonoaudiólogos, devem verificar se seus pacientes utilizam recursos da internet, reconhecer esta mudança de comportamento e preparar-se não só para discutir as informações obtidas com o paciente, mas também sugerir websites com informações confiáveis e auxiliá-los a avaliar a qualidade das informações disponíveis online.

Palavras-chave: aconselhamento, internet, fonoaudiologia.

\section{SUMMARY}

Introduction: The process of education of a patient aims to improve knowledge and skill of the patient and/or family, in a way to influence attitudes and behaviors needed to maintain or improve health. Must be a integral part of interpersonal communication between health professionals and patients, and this can happen through interpersonal communication and various other means such as pamphlets, manuals and, more recently, computer resources.

Goal: This update article approach the patient education and the internet potential for this process, still presenting some initiatives in the audiology área.

Discussion: The informations, related to health are popular in the internet, and include interactive websites, portals, emails, telehealth aplications, and others. The education of the patients supported by the internet can help to solve a big ethical, political and economic issue: the problem to conciliate the needs and the expectations of the patients with the characteristics and limitations of the health system. Although the use of internet in the education of the patient is promising, this is not a solution to be used without careful planning, monitoring and evaluation. In audiology there are few initiatives with the use of e-mails and websites for complement of the patient education.

Final Considerations: The health professionals, including the speech therapists, must check if their patients use internet resources, recognize that behavior change and prepare not only to discuss the information obtained with the patient, but also suggest websites with reliable information and help them to evaluate the quality of the information available online.

Keywords: counseling, internet, speech language pathology. 


\section{INTRODUÇÃO}

Nas décadas de sessenta e setenta os profissionais da saúde eram responsáveis pelo diagnóstico, tratamento e acompanhamento dos pacientes, sendo estes últimos considerados participantes passivos no processo de diagnóstico e tomada de decisões no tratamento. A partir da década de oitenta vários países estabeleceram legislações que davam direito ao paciente de saber sobre sua condição de saúde e opinar sobre o tratamento. Nos anos noventa os pacientes passaram a ser ativamente envolvidos na promoção de sua saúde, partilhando com os profissionais de saúde a responsabilidade por decisões de tratamento e seu objetivo, em um diálogo mais igualitário em lugar de um modelo paternalista. Além disto, a influência de pessoas do convívio do paciente como cônjuges, filhos e outros membros de sua rede social, foi reconhecida como importante no processo interventivo, sendo até mesmo incorporada como um sistema de apoio. Nesta época, ressaltou-se de forma mais contundente a importância dos comportamentos auto-regulatórios do paciente, em sua vida diária, nos resultados do tratamento o que, por conseguinte, levou a uma maior preocupação com o processo de educação ao paciente (1).

Neste contexto, a educação ao paciente é entendida como parte do tratamento que permite uma revisão crítica do tradicional relacionamento profissional-paciente, enfocando os aspectos pessoais e emocionais e o reconhecimento da necessidade de centralizar a pessoa e não a doença (2). O objetivo principal é permitir ao paciente realizar escolhas conscientes sobre o gerenciamento de sua condição (3).

De alguma forma, todos os profissionais da saúde fornecem aos pacientes e/ou familiares informações sobre suas condições e tratamento, prevenção secundária e possíveis complicações. Isto pode ocorrer por meio da comunicação interpessoal e/ou diferentes meios como panfletos, manuais e, mais recentemente, recursos computacionais.

Os avanços nas tecnologias da informação, computação e comunicação transformaram a vida econômica, social e cultural da sociedade. Os computadores, mais do que ferramentas que facilitam a vida das pessoas, tornaramse parte da cultura (4). Os computadores e a internet possibilitam a obtenção de informação em diferentes fontes, locais, horários e em uma velocidade sem precedentes, daí o seu grande impacto no processo de educação ao paciente. Este artigo de atualização aborda a educação ao paciente e as potencialidades da internet para este processo, apresentando ainda, algumas iniciativas na área da Audiologia.

\section{REVISÃO DE LITERATURA}

A atividade de educação ao paciente tem recebido diferentes denominações, não havendo uma terminologia consistentemente empregada na literatura fonoaudiológica ou mesmo em outras áreas da saúde. Muitas vezes os termos aconselhamento, orientação e educação ao paciente são empregados como sinônimos. Este cenário pode levar a compreensões equivocadas das práticas profissionais.

O aconselhamento é uma oportunidade de receber e fornecer informações de modo a facilitar o entendimento de uma condição de saúde e propiciar ajuste a esta situação. Dois tipos de aconselhamentos são definidos na literatura: (a) aconselhamento de ajuste pessoal, que envolve o desenvolvimento de mecanismos e sistemas de suporte emocional para o indivíduo lidar com a situação, e (b) aconselhamento informativo, que aborda questões relacionadas a uma determinada doença, fisiologia normal, resultados de exames e plano de tratamento (5). O termo "orientação" é frequentemente utilizado no lugar de "aconselhamento informativo".

O Conselho Federal de Fonoaudiologia utiliza os termos "orientação" e "aconselhamento" para se referir a uma das áreas de competência do fonoaudiólogo que engloba ações de escuta profissional, a explicação, instrução, demonstração, proposição de alternativas e verificação da eficácia das ações propostas e da compreensão das orientações ministradas (6).

O termo "educação ao paciente" refere-se a todas as atividades educacionais dirigidas aos mesmos, incluindo aspectos de educação terapêutica, educação em saúde e promoção de saúde. O processo de educação ao paciente tem como objetivos melhorar o conhecimento e habilidade do paciente e/ou família de forma a influenciar as atitudes e comportamentos necessários para manter ou melhorar a saúde (7).

A educação terapêutica é definida como o processo de auxiliar o paciente a adquirir ou manter as competências necessárias para lidar da melhor forma possível com uma doença em sua vida. É, portanto, um processo contínuo, integrado com o cuidado à saúde. É centrada no paciente e inclui atenção, informação, aprendizagem de autocuidado e suporte psicossocial sobre a doença, tratamento prescrito, sobre o hospital ou outras instituições de saúde, informação organizacional e de comportamento relacionadoà saúde e à doença (8).

O surgimento da educação terapêutica como área de estudo indica os sinais do declínio do modelo biomédico 
reducionista e dualista em favor de um modelo biopsicossocial com uma perspectiva sistêmica. Atençãoé dada ao conceito de saúde e não mais ao de doença, deslocando o eixo em torno do qual gira a intervenção clínica, da doença para o bem-estar. O protagonista é o próprio indivíduo que age para melhorar sua saúde (2). Por pressupor o paciente como agente ativo no processo, o termo "educação ao paciente" é empregado neste artigo.

Os benefícios da educação ao paciente podem incluir alguns ou todos os itens a seguir: maior conhecimento da doença e compreensão das circunstâncias desta para cada paciente, melhor compreensão da necessidade de tratamento, melhor comunicação entre o paciente e o profissional de saúde, participação dos pacientes na escolha do tratamento, desenvolvimento de opiniões e modelos adequados sobre a doença, melhoria do autocuidado, melhoria no gerenciamento de crises, desenvolvimento de habilidades de enfrentamento e de solução de problemas, bem-estar emocional, melhora da qualidade de vida, maior consciência da importância de consultas de acompanhamento e/ou necessidade de mais procedimentos de triagem e diagnóstico, maior concordância com os planos de tratamento, melhor uso de tempo e dos recursos (3).

Algumas doenças crônicas levantam questões em relação ao significado do estado de saúde para os pacientes e familiares. Pode haver importantes consequências no modo como lidam com a vida diária como, por exemplo, a necessidade de ingestão de medicamentos, uso de próteses ou realização de exames de saúde periódicos. Estes fatores levam o paciente a uma reestruturação cognitiva da percepção de si mesmo, um "eu" que pode ter sofrido consideráveis modificações físicas e a organização de uma nova identidade, reconhecendo o estado de "estar doente". Assim a educação ao paciente também é um instrumento terapêutico e requer profunda sensibilidade relacionada aos aspectos de relações interpessoais, necessidades individuais, expectativas e reais possibilidades de recuperação e reabilitação (2).

Destaca-se, no entanto, que nem sempre as informações consideradas essenciais pelos profissionais são aquelas que o paciente considera necessárias (9). Deste modo, é importante que o profissional reflita sobre suas atitudes e opiniões sobre o conhecimento, aprendizado e educação como parte do tratamento do paciente. "O paciente é tratado como um parceiro no aprendizado ou como um aluno? Há um diálogo com o paciente para se saber as necessidades de aprendizagem e ideias ou é apenas uma comunicação de mão única com material padrão?" Não há aprendizado sem reflexão, interação, compreensão e interpretação eficientes por parte do paciente (10).
O processo de educação ao paciente pode ser descrito em cinco passos. O primeiro inclui a avaliação do conhecimento prévio que o paciente possui, equívocos, habilidades e estilos de aprendizagem, cognição, atitudes e motivação. Isso pode ser recolhido por meio de entrevista ou testes específicos. Após esta avaliação, os recursos dos pacientes, as barreiras e as necessidades de aprendizado podem ser identificadas. O terceiro passo é o planejamento da educação juntamente com o paciente, os objetivos são definidos e as intervenções são escolhidas. Na fase de planejamento o método utilizado para a educação, quem irá fornecê-la, a frequência, como e quando a educação irá ocorrer devem ser definidos. O próximo passo é o fornecimento da educação, e o último a avaliação. A partir disto, a avaliação continuada das necessidades do paciente e seus objetivos fornecem a base para mais educação (11).

Olocal escolhido para a educação ao paciente pode ser um hospital, um ambulatório, unidades de atenção primária, a residência do paciente ou mesmo a combinação de tais locais. O processo de educação inicia-se na atenção primária ou no hospital, dependendo de onde o paciente foi diagnosticado. Uma vez que o paciente recebe educação em diferentes momentos e de diferentes profissionais, o conteúdo deve ser consistente nesta cadeia de atendimentos, ou seja, as informações devem ser claras e não contraditórias a fim de conseguir uma maior concordância do paciente ao tratamento (10).

As tecnologias de informação e comunicação estão presentes na maioria dos campos da atividade humana, incluindo a área da saúde. A disseminação do uso da internet vem proporcionando um crescimento acentuado do acesso à informação com benefícios para a educação ao paciente.

A internet é uma rede global de computadores interligados por um protocolo específico que permite não só o acesso às informações, mas também a transferência de dados de um computador a outro. Em dezembro de 2010 cerca de 1,9 bilhões de pessoas possuíam acesso à internet, uma penetração global de aproximadamente $29 \%$. A razão de usuários no mundo cresceu $445 \%$ entre o ano 2000 e 2010. A América Latina e Caribe contam com 200,1 milhões de usuários sendo 75,9 milhões de pontos existentes só no Brasil (12).

O Centro de Estudos sobre as Tecnologias da Informação e da Comunicação no Brasil entrevistou 19.998 pessoas das áreas urbanas e rurais no período de setembro e novembro de 2009 , coletando informações sobre o uso da internet. A porcentagem de indivíduos com acesso à internet neste período encontra-se na Figura 1 (13). 


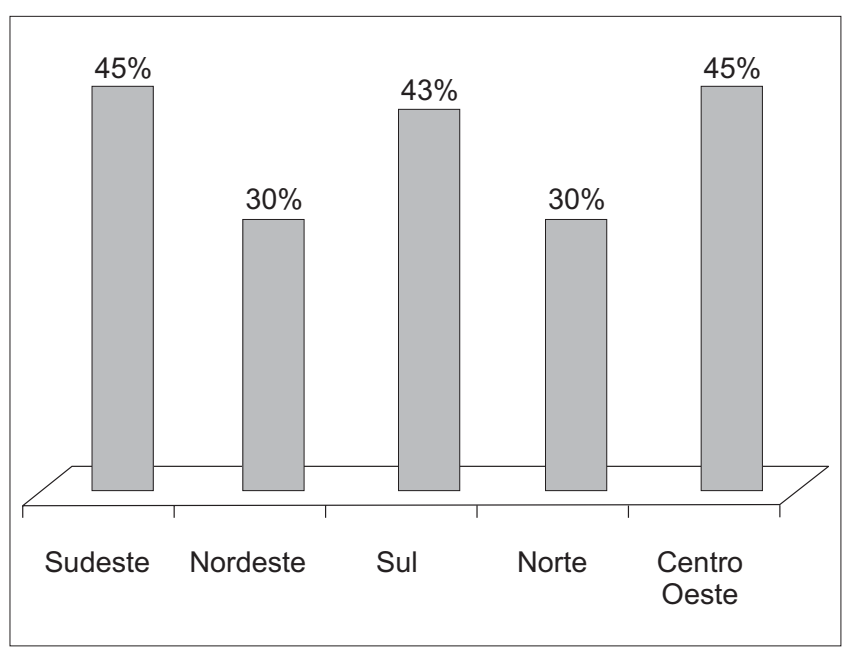

Figura 1. Porcentagem de usuários de internet por regiões do Brasil (13).

As informações relacionadas à saúde são populares na internet, e incluem websites interativos, portais, e-mails, aplicações de telessaúde, comunidades online, jogos e simuladores. Os websites com informações de saúde são um dos mais visitados - isto significa que os pacientes estão coletando informações fora da interação tradicional com o profissional de saúde (1). Existe desta forma a descentralização e democratização do acesso à informação, com a possibilidade da informação ser revista quantas vezes o paciente julgar necessário.

A internet também proporciona uma fonte útil de informação e apoio para uma ampla gama de condições crônicas, proporciona fóruns convenientes para muitas pessoas, evitando vários obstáculos convencionais para apoiar a participação de grupos como, por exemplo, tempo, localização geográfica, barreiras pessoais e físicas (3). A educação de pacientes apoiada na internet pode contribuir para solucionar um grande desafio ético, político e econômico: o problema de conciliar as necessidades e expectativas dos pacientes com as características e limitações do sistema de saúde.

Computadores podem auxiliar no processo de democratização e disseminação de informações sobre a saúde e permitir que, através deste conhecimento, indivíduos, famílias e comunidades assumam um papel mais ativo nos cuidados com a própria saúde, minimizando a carga psicológica frente a tratamentos, exames ou cirurgias, bem como diminuindo o uso de serviços onerosos e desnecessários (14).

Uma revisão sistemática com doze estudos sobre as experiências subjetivas de pacientes que utilizaram serviços de comunicação interativa de saúde mostrou que o uso de tais aplicações na internet aumentou o conhecimento, a confiança e melhoraram a saúde de tais pacientes. Isto, por sua vez, permitiu que fossem estreitadas as relações paciente-profissional de saúde (15).

Com relação aos hábitos de pacientes brasileiros em relação ao uso da internet, 83\% procuram informações sobre saúde na web e $85 \%$ voltam a fazer pesquisas on-line depois de uma consulta médica. Os pacientes revelaram que as informações obtidas na internet eram úteis e os tornavam mais ativos em relação às decisões sobre sua saúde. Mais que 50\% destes indivíduos que fazem buscas por informações de saúde na internet utilizam o Sistema Único de Saúde (16).

A popularidade e o aumento de informações de saúde disponíveis na internet apresentam novos desafios, como, por exemplo, a qualidade e a objetividade das informações a presentadas. Vários instrumentos de avaliação têm sido desenvolvidos com a finalidade de guiar os consumidores para boas fontes de informação. Estes instrumentos são geralmente genéricos de forma que podem ser aplicados para websites que fornecem diferentes informações para uma série de condições de saúde. A mensuração do impacto clínico e a capacidade do consumidor aprender o conteúdo encontrado online também devem ser consideradas na avaliação de um website (17).

$\mathrm{Na}$ Alemanha foi observado como as pessoas realizavam buscas na internet sobre assuntos relacionados à saúde e como avaliavam as informações encontradas. A maioria dos indivíduos utilizava buscadores como, por exemplo, o Google. Foi observado que 97,2\% analisavam apenas os 10 primeiros resultados e depois realizavam uma nova busca. Os participantes utilizavam os seguintes critérios para avaliar a qualidade dos websites: origem das informações, aparência e layout do website, facilidade de leitura, existência de links, contato fornecido, qualificações dos autores, atualizações do conteúdo e selo de qualidade fornecido por algum órgão responsável (18).

Um dos instrumentos mais populares para avaliação de websites é o Health on the Net Code (HONcode), desenvolvido por um grupo de profissionais da saúde que observaram a necessidade de avaliação da qualidade das informações de saúde fornecidas online. O HONcode certifica websites que obedeçam a oito critérios (19):

- Qualificações dos autores: qualquer orientação de saúde oferecida no website deverá ser fornecida por profissionais treinados e qualificados.

- Complementaridade: as informações oferecidas devem auxiliar, e não substituir, a relação médico-paciente.

- Privacidade: devem ser respeitadas a privacidade e confidencialidade dos dados pessoais que o usuário ou visitante envia para o website de acordo com os requisitos legais do estado ou país onde o website foi desenvolvido. 
- Atribuição: devem ser citados a bibliografia consultada e/ou as fontes dos dados fornecidos no website e, se houver, devem ser fornecidos os links para tais fontes. A data em que cada página foi modificada pela última vez deverá ser fornecida de maneira clara.

- Justificativas: quaisquer afirmações feitas sobre os benefícios e desempenho de um determinado tratamento, produto comercial ou serviço deverão ser sustentados por evidências.

- Transparência: a informação deve ser apresentada de forma acessível. Deve ser oferecida uma forma de contato para os visitantes que desejarem informações ou ajuda adicional.

- Patrocínio: as fontes de financiamento devem estar identificadas.

- Política de Publicidade: uma breve descrição da política de divulgação adotada pelo website deve ser apresentada.

O Centro Nacional para Medicina Complementar e Alternativa (20) também fornece um guia para os usuários da internet avaliarem websites com informações de saúde de acordo com dez perguntas básicas: 1.Quemé o responsável pelo site e pelas informações que estão nele? 2. Quem financia o site? 3. Qual é o propósito/objetivo do site? 4. De onde vem a informação fornecida no site? 5 . Quais são as referências das informações? 6. Como foi selecionada a informação? Ou seja, há corpo editorial? O conteúdo foi revisado por profissionais com qualificações científicas? 7. Quão atualizadas são as informações? 8. Como este site escolhe links para outros sites? (alguns sites pagam para terem seus links em outras páginas; outros estabelecem alguns critérios para colocar links). 9. Qual informação sobre o visitante que foi coletada pelo website e por quê? 10. Como o site administra interações com seus visitantes/usuários?

Um estudo comparou a usabilidade de diferentes instrumentos para avaliação de websites de saúde. As pesquisadoras solicitaram que cinco participantes navegassem no website "Saúde na Internet" e avaliassem via administração de três instrumentos diferentes traduzidos para o português pelas autoras: (a) Emory (22), 36 itens divididos nas escalas conteúdo, precisão, autores, atualizações, público, navegação, links e estrutura - apresenta fácil pontuação e preenchimento; (b) Michigan (22), 43 itens divididos nas escalas conteúdo, precisão, autores, atualizações, público, valores, navegação, links, publicidade e estrutura - apresenta fácil entendimento, porém, é de difícil pontuação; e (c) o HonCode adaptado para o português (23). Foram avaliados o tempo despendido e resultado obtido pela aplicação de cada instrumento. Os comentários dos participantes sobre o uso dos instrumentos foram analisados qualitativamente. O tempo médio de aplicação dos instrumentos foi de 2,2 (HonCode), 11 (Emory) e 13 minutos (Michigan). Para cada instrumento utilizado obser- vou-se variabilidade da pontuação interavaliadores, sendo esta maior para o questionário Michigan. Houve discrepância do resultado da avaliação interinstrumentos, ou seja, o website foi considerado adequado pelos instrumentos Emory e HonCode e fraco pelo Michigan. A classificação obtida pela aplicação do instrumento nem sempre correspondeu ao julgamento subjetivo da qualidade do website dado pelo avaliador, especialmente no caso do HonCode. O questionário Michigan foi considerado de difícil entendimento e muito longo. O Emory foi considerado o mais fiel na classificação do website e o de maior facilidade de entendimento das questões (24).

Para a construção de sistemas de informação computadorizados faz-se necessário seguir atributos de qualidade para que o mesmo obtenha bons resultados em sua avaliação. O desenvolvimento de um website contendo informações de saúde pode seguir os atributos de qualidade de um sistema de informação computadorizado (25), sendo eles:

- Usabilidade: considera as diferentes formas de sua utilização, durante as fases de desenvolvimento e uso. Para os desenvolvedores deve se garantir que as atividades relacionadas ao desenvolvimento e manutenção possam ser realizadas com qualidade e facilidade. Para os profissionais da saúde, garantir que o sistema possua características adequadas aos seus usuários (os pacientes) e que, ao surgirem necessidades de alterações e/ou de introdução de novas informações, estas poderão ser realizadas num tempo hábil e com custo aceitável, mantendo-se a qualidade desejada. Para o usuário final (os pacientes) os atributos de qualidade relacionados a este objetivo referem-se a características relacionadas à facilidade de uso do sistema eà satisfação do usuário com o seu uso.

- Confiabilidade conceitual: avaliar e garantir a confiabilidade do conteúdo disponível. As informações fornecidas não podem apresentar erros, pois, do contrário, podem levar a consequências indesejadas para a saúde dos usuários finais - os pacientes.

- Confiabilidade de representação: para que um sistema de educação ao paciente atenda o objetivo de complementar ou, em casos específicos, substituir as informações dadas por profissionais de saúde é necessário que estas estejam representadas de forma a facilitar seu entendimento.

Uma revisão literária identificou quatro pontos principais para que o uso da tecnologia de informação e comunicação atinja seu potencial pleno para a educação ao paciente. As intervenções baseadas na internet devem ser desenvolvidas de modo a maximizar a comunicação interativa com os usuários de forma a encorajar seu envolvimento ativo na promoção e cuidado à saúde. Os sistemas devem ser desenhados para trabalhar de forma 
efetiva e transparente em diferentes plataformas de comunicação e com diferentes populações de usuários. As intervenções devem ser desenhadas para engajar os interesses e emoções de usuários para promover a máxima exposição e influência das informações postadas. Finalmente, as aplicações devem ser desenvolvidas para ter um acesso amplo a diversas populações e, ao mesmo tempo, adaptar-se as necessidades e interesses específicos de diferentes usuários (26).

\section{DISCUSSÃO}

Embora a utilização da internet para a educação ao paciente seja promissora, esta não é uma solução que deve serutilizada sem planejamento cuidadoso, monitoramentoe avaliação. A tecnologia deve ser utilizada como um meio para se chegar a uma finalidade e não como a finalidade em si. Os cinco passos do processo de educação descritos por Rankin e Stallings (11) podem ser aplicados quando se planeja um material de educação ao paciente baseado na internet.

Uma das primeiras considerações sobre o uso da internet no processo de educação ao paciente diz respeito ao acesso do mesmo à internet bem como aos custos envolvidos. É importante saber se o paciente possui ou tem acesso a um computador, se sabe como usá-lo e se tem interesse em utilizar este recurso para obter informações de saúde. Embora o uso da internet continue aumentando, existe uma lacuna entre as pessoas com acesso efetivo às tecnologias digitais e de informação e aqueles com pouco ou nenhum acesso - neste último caso pessoas com menor instrução, menor renda familiar, com idade superior a 65 anos e que vivam em áreas rurais (4).

Outra questão de grande importância é o controle muito limitado das informações que são inseridas na internet. A preocupação de muitos profissionais é que os pacientes tomem importantes decisões sobre sua saúde baseados em informações que não tenham sido revisadas em termos de sua qualidade, objetividade, atualidade, acurácia e ausência de viés. Ressalta-se que pode haver outros interesses, como por exemplo, econômicos, por trás das informações fornecidas (7) daí a importância de avaliação dos websites.

A avaliação dos websites de saúde traz vários benefícios, entre eles, promoção de melhorias na qualidade, utilidade e efetividade do website, diminuição de possíveis efeitos negativos, conservação de recursos e participação de potenciais usuários no processo de desenvolvimento do website. As avaliações podem ser realizadas antes do desenvolvimento do website, durante e após a divulgação do mesmo, garantindo constantes atualizações de conteúdo e aprimoramento da apresentação (27).
Além da avaliação da qualidade do conteúdo são de extrema importância a mensuração do impacto clínico e da capacidade do próprio consumidor aprender o conteúdo encontrado online (17).

Nem todas as informações existentes na web são de fácil compreensão. Além disto, pode ser que o número de informações existentes seja maior do que aquela com que o indivíduo consiga lidar. Por exemplo, um indivíduo em fase inicial de diagnóstico pode encontrar informações sobre o curso da patologia. Desta forma é importante que o profissional pergunte ao paciente se estes têm utilizado a internet e explorar os tipos de informações encontradas. Alguns pacientes não se sentem à vontade em revelar que procuram informações na internet com receio de que isto abale a relação entre ele e o profissional, ou seja, que o profissional entenda isto como falta de confiança no cuidado recebido.

Muitos pacientes realizam buscas na internet sobre suas condições de saúde e de membros de sua família mesmo antes de procurar o profissional de saúde. Desta forma, os pacientes terão noções prévias, verdadeiras ou não, sobre o diagnóstico e tratamento. Desta forma, faz-se necessário uma mudança na conduta do profissional para lidar com estes pacientes, assumindo uma postura mais aberta, capaz de trabalhar as informações encontradas pelos pacientes e indicar as fontes com maior fidelidade, deixando assim a antiga postura autoritária (28).

Foi realizada uma revisão dos estudos examinando como as buscas na internet feitas pelos pacientes afetavam as relações com os seus profissionais de saúde. De modo geral, três tipos de resultados foram encontrados: (a) os profissionais se sentiram ameaçados por pacientes que trazem informações obtidas na internet e reagem defensivamente oferecendo sua "opinião de especialista"; (b) o profissional e o paciente atuam em conjunto para encontrar e analisar a informação e (c) o profissional guia o paciente para websites que sejam confiáveis (29).

O uso da internet como ferramenta para auxiliar o processo de educação ao paciente vem sendo discutido em diferentes áreas da saúde, sobretudo na enfermagem, medicina, psicologia, nutrição e farmácia tanto nacional como internacionalmente. No que tange a fonoaudiologia, existe na internet um grande número de websites com informações referentes aos diversos processos e distúrbios da comunicação sendo estes desenvolvidos tanto por sociedades científicas como por empresas ou clínicas que atuam na área. No entanto, até o presente momento, estudos que avaliaram a qualidade, conteúdo e uso de tais websites para a educação do paciente são extremamente escassos.

KALDO-SANDSTRÖm, LARSEn e ANDERSSOn (30) avaliaram a efetividade da terapia de cognição-comportamento aplica- 
da por meio de um website no tratamento do zumbido. Os participantes completaram módulos semanais e a o final de cada sessão completavam um diário no próprio website para monitoramento do progresso. Foi observada significativa redução do estresse, ansiedade, depressão e insônia para aqueles que cumpriram o programa. O nível de desistência dos participantes foi de 30\% sendo esta maior do que a observada para o tratamento face a face. No entanto, não houve diferença entre os julgamentos de credibilidade do tratamento aplicado tradicionalmente e pela internet.

NoBrasil pesquisadoras avaliarama eficácia do website "Portal dos Bebês - Fonoaudiologia" como instrumento de orientação à distância aos pais de crianças deficientes auditivas usuárias de AASI em um estudo preliminar. Neste estudo participaram 31 pais de crianças deficientes auditivas recém adaptadas com AASI, divididos em grupos $\mathrm{A}(\mathrm{n}=18)$ e $B(n=13)$ que receberam orientações presenciais sobre as características da deficiência auditiva e uso e cuidados com o AASI. O grupo B acessou o website durante os meses iniciais douso do AASI. Foi aplicadoum questionário fechado a respeito das orientações fornecidas imediatamente após a adaptação do AASI e no primeiro retorno da criança. Ambos os grupos reconheceram 83\% da informação sobre as características da perda auditiva no momento da adaptação e no retorno. Quanto ao uso e cuidados com o AASI os participantes reconheceram 89\% (A) e 85\% (B) da informação na adaptação e 77\% (A) e 94\% (B) no retorno. Na avaliação realizada no retorno o grupo $\mathrm{B}$ reconheceu significativa maior quantidade de informação que o grupo A. As autoras puderam concluir que programas de orientação a pais de crianças deficientes auditivas que permitam o acesso conveniente às orientações fornecidas são necessários para auxiliá-los na retenção da informaçãoe, consequentemente, assegurar o uso efetivo do AASI (31).

\section{COMENTÁRIOS FINAIS}

O uso da internet como fonte de informação de saúde vem se tornando cada vez mais popular. Os profissionais da saúde, incluindo os fonoaudiólogos, devem verificar se seus pacientes utilizam tal recurso, reconhecer esta mudança de comportamento e preparar-se não só para discutir tais informações com o paciente, mas também sugerir websites com informações confiáveis e auxiliá-los a avaliar a qualidade das informações disponíveis online.

Também é de importância que fonoaudiólogos participem mais ativamente no desenvolvimento de websites que auxiliem o processo de educação do paciente tendo como base para a criação de conteúdos as necessidades e perspectivas dos usuários, os princípios de qualidade bem como as possibilidades de interação oferecidas pela internet 2.0 ou web 2.0. Este termo identifica a mudança para uma Internet como plataforma, onde os aplicativos aproveitam os efeitos de rede para se tornarem melhores quanto mais são usados pelas pessoas, aproveitando a "inteligência coletiva". O usuário é ativo, ou seja, não só usufrui, mas contribui. O exemplo clássico da web 2.0 é o Wikipedia onde cada usuário tem a possibilidade de ler e adicionar informações livremente.

Os indivíduos que buscam informações de saúde na internet podem utilizar estas mesmas ferramentas da web 2.0 para compartilhar não só informações, mas, sobretudo, seus sentimentos e experiências do "estar doente", para, desta forma, darem significadoa o momento conturbado que vivenciam; para compartilhar suas experiências, suas angústias e seus sofrimentos do adoecer com outras pessoas, e para construírem conhecimentos sobre seus problemas de saúde, permitindo se sentirem mais seguros e confiantes durante o tratamento ao qual são submetidos (32).

Finalmente deve ser lembrada que a educação ao paciente baseada na internet é ainda um campo bastante novo de pesquisa para a Fonoaudiologia, devendo a mesma ser utilizada como forma de complementação e não de substituição da educação fornecida de forma presencial. É imprescindível a realização de estudos randomizados e controlados a fim de avaliar a efetividade e eficácia dos processos de educação ao paciente via internet.

\section{REFERÊNCIAS BIBLIOGRÁFICAS}

1.Hoving C, Visser A, Mullen PD, Van Den Borne B. A history of patient education by health professionals in Europe and North America: From authority to shared decision making education. Patient Educ Couns. 2010; 78: 275-81.

2. WorldHealth Organization. Therapeutic patient education of patients with coronary heart disease: training guide for general practioners. Udine (Italy): WHO, 2006.

3. Reed K. Therapeutic Patient Education. Disponível em: http://www.diabetesinfo.org.nz/library/pe_therapeutic.pdf. Acesso em: 14 Jun. 2010.

4. Sopeczik DL. Technology in education. In: Bastable SB. Essentials of patient education. Sudbury: Jones and Bartlett, 2006. Cap. 13, p. 502p.

5. Margolis RH. Page 10: What do your patients remember? Hear J. 2004, 7:10-7.

6. Conselho Federal de Fonoaudiologia. Exercício do profissional fonoaudiólogo. 2002. Disponível em: http:// www.fonoaudiologia.org.br/publicacoes/epdo1.pdf.Acesso em: 01 Fev. 2011. 
7. Visser A, Deccache A, Bensing J.Patienteducation in Europe: united differences. Patient Educ Couns. 2001, 44: 1-5.

8. World Health Organization. The therapeutic patient education: continuing education programmes for health care providers in the field of prevention of chronic diseases. Copenhagen (Denmark): WHO, 1998.

9. Falvo D. Effective patient education: a guide to increased compliance. 3. ed. Sudbury: Jones andBarlettPublishers; 2004.

10. Strömberg A. The crucial role of patient education in heart failure. Eur J Heart Fail. 2005; 7(3): 363-9. Disponível em: <http://www.bmj.com/cgi/reprint/324/7337/573>. Acessado em: 13 out. 2008.

11. Rankin SH, Stallings KD. Patient Education: Principles and Practice. 4 a ed. Philadelphia: Lippincott, Williams, \& Wilkins; 2001.

12. Internet World Stats. Usage and population statistics. 2009. Disponível em: <http://www.internetworldstats.com/ stats.htm>. Acesso em: 10 apr. 2010.

13. Centro de Estudos de Sobre Tecnologias da Informação e da Comunicação (CETIC). Pesquisa sobre o uso das tecnologias da informação e comunicação no Brasil: TIC domicúlios e usuários 2009. Disponível em: http:// www.cetic.br/usuarios/tic/2009-total-brasil/rel-int-02.htm. Acesso em: 27 de janeiro de 2011.

14. Abramson R. Personal physician: an expert system designed for patients. MD Comput. 1995, 12:11-3.

15. Âkesson KM, Saveman BI, Nilsson G. Health care consumers experiences of information communication technology - a summary of literature. Int J Med Inform. 2007, 76:633-45.

16. Silva WM. Navegaré preciso: avaliação de impactos do uso da internet na relação médico-paciente. São Paulo, 2006, p.146(Dissertação de Mestrado-Faculdade de SaúdePública da Universidade de São Paulo).

17. O'Grady L. Future directions for depicting credibility in health care web sites. Int J Med Inform. 2006, 75:58-65.

18. Eysenbach G, Köhler C. How do consumers search for and appraise health information on the world wide web? Qualitative study using focus groups, usability tests, and indepth interviews. BMJ. 2002, 324:573-7.

19. Health on the Net Foundation. Disponível em: Acesso em: 24 Oct. 2008.
20. National Centre for Complementary and Alternative Medicine (NCCAM). 10 Things to Know About Evaluating Medical Resources on the Web. 2006. Disponível em: Acesso em: 21 Oct. 2008.

21. Emory University Rollins School of Public Health. HealthRelated Web Site Evaluation Form. 1998. Disponível em: http://www.sph.emory.edu/WELLNESS/instrument.html. Acesso em: 10 abr. 2009.

22. Anderson NA, Groove S, Hill S. Web Site Evaluation Checklist 1999, 1998. University of Michigan.

23. Barbosa AL, Martins EN. Avaliação da informação dos pacientes sobre miopsias e fotopsias através da Internet. Arq Bras Oftalmol. 2007, 70:839-43.

24. Souza PJS, Bastos BG, Ferrari DV. Instrumentos para avaliação de websites de saúde: estudo preliminar. Trabalho apresentado na XVI Jornada Fonoaudiológica de Bauru, Bauru, Ago. 2009.

25. Valle C, Ximenes AA, Campos GHB, Rocha AR, Rabelo A. Educação de Pacientes através de Sistemas de Acesso Público. RBIE. 1997, 1.

26. Kreps GL, Neuhauser L. New directions in eHealth communication: Opportunities and challenges. Patient Educ Couns. 2010, 78:329-36.

27. Eng TR, Gustafson DH, Henderson J, Jimison H, Patrick K. Introduction to evaluation of interactive health communication applications. Am J Prev Med. 1999, 16:10-5.

28. Kuppersmith RB. The physician-patient relationship and the internet. Otolaryngol Clin North Am. 2002, 35:1143-7.

29. Mcmullan M. Patients using the Internet to obtain health information: how this affects the patient-health professional relationship. Patient Educ Couns. 2006, 63:24-8.

30. Kaldo-Sandström V, Larsen HC, Andersson G. Internetbased cognitive-behavioral self-help treatment of tinnitus: clinical effectiveness and predictors of outcome. AmJ Audiol. 2004, 13:185-92.

31. Ferrari DV, Tomé T, Bastos BG. Internet based hearing aid orientation. Trabalho apresentado no British Academy of Audiology Convention, Liverpool, 2008.

32. Maia F, Struchiner M. The use of weblogs and orkut communities as pedagogical tools in courses in the health area. Interface Comum Saúde Educ. 2010, 14:905-18. 